\title{
Clinical predictors of genetic testing outcomes in hypertrophic cardiomyopathy
}

\author{
Jodie Ingles, BBiomedSci, PhD ${ }^{1,2}$, Tanya Sarina, MACommsMgmt ${ }^{1}$, Laura Yeates, BSc', \\ Lauren Hunt, BSc, MSc ${ }^{3}$, Ivan Macciocca, BSc, MSc ${ }^{4,5}$, Louise McCormack, MBBS ${ }^{6}$, \\ Ingrid Winship, MBChB, MD5, Julie McGaughran, MBChB, $\mathrm{MD}^{3,7}$, John Atherton, $\mathrm{MBBS} \mathrm{PhD}^{6,7}$ and \\ Christopher Semsarian, MBBS, PhD ${ }^{1,2,8}$
}

\begin{abstract}
Purpose: Genetic testing for hypertrophic cardiomyopathy has been commercially available for almost a decade; however, low mutation detection rate and cost have hindered uptake. This study sought to identify clinical variables that can predict probands with hypertrophic cardiomyopathy in whom a pathogenic mutation will be identified.
\end{abstract}

Methods: Probands attending specialized cardiac genetic clinics across Australia over a 10-year period (2002-2011), who met clinical diagnostic criteria for hypertrophic cardiomyopathy and who underwent genetic testing for hypertrophic cardiomyopathy were included. Clinical, family history, and genotype information were collected.

Results: A total of 265 unrelated individuals with hypertrophic cardiomyopathy were included, with 138 (52\%) having at least one mutation identified. The mutation detection rate was significantly higher in the probands with hypertrophic cardiomyopathy with an established family history of disease ( 72 vs. $29 \%, P<0.0001$ ), and a positive family history of sudden cardiac death further increased the detection rate ( 89 vs. $59 \%, P<0.0001)$. Multivariate analysis identified female gender, increased left-ventricular wall thickness, family history of hypertrophic cardiomyopathy, and family history of sudden cardiac death as being associated with greatest chance of identifying a gene mutation. Multiple mutation carriers $(n=16,6 \%)$ were more likely to have suffered an out-of-hospital cardiac arrest or sudden cardiac death ( 31 vs. $7 \%, P=0.012$ ).

Conclusion: Family history is a key clinical predictor of a positive genetic diagnosis and has direct clinical relevance, particularly in the pretest genetic counseling setting.

Genet Med advance online publication 18 April 2013

Key Words: genetic counseling; genetic testing; hypertrophic cardiomyopathy
Hypertrophic cardiomyopathy (HCM) is an inherited cardiomyopathy characterized by myocardial hypertrophy, usually of the left ventricle, in the absence of other loading conditions such as hypertension. ${ }^{1,2}$ HCM affects 1 in 500 of the general population and is an important cause of sudden cardiac death (SCD) in the young, particularly in athletes. ${ }^{3,4}$ HCM shows extreme clinical heterogeneity, with many individuals experiencing minimal or no symptoms whereas others may suffer the most severe outcomes of heart failure and SCD.

$\mathrm{HCM}$ is inherited in an autosomal dominant manner. Mutations in at least 13 sarcomere or sarcomere-related genes have been shown to cause HCM. Commercial genetic testing is widely advocated as a necessary component of management, allowing clarification of the genetic status of asymptomatic at-risk relatives. ${ }^{5}$ Despite HCM proband genetic testing being available commercially for almost 10 years, there are still a number of limitations to this testing process. Specifically, the quoted mutation detection rates vary substantially, between 20 and $63 \%,{ }^{6-8}$ meaning a significant number of probands will undergo this expensive testing procedure and have no causative mutation identified (i.e., an indeterminate genetic result). ${ }^{7,9,10}$ In cases in which an indeterminate genetic result is given, the asymptomatic family members must continue with ongoing periodic clinical surveillance strategies, which will fail to detect those patients without an obvious phenotype. ${ }^{11,12}$ In addition, the cost of proband genetic testing for HCM has been a prohibitive factor that has limited uptake, despite it being shown to be a cost-effective addition to conventional HCM management in the long term. ${ }^{13,14}$

One explanation for the variable genetic testing detection rates may relate to the clinical phenotype and family history of those undergoing genetic testing. ${ }^{7,15}$ A number of HCM phenocopies and genocopies exist, such as glycogen storage diseases, which may influence the mutation detection rate of $\mathrm{HCM}$ genetic testing, as might the presence or absence of a family history of HCM. A simple method of identifying probands with HCM who are more likely to have a gene mutation identified would be of significant benefit, both from a clinical

\footnotetext{
${ }^{1}$ Agnes Ginges Centre for Molecular Cardiology, Centenary Institute, Sydney, Australia; ${ }^{2}$ School of Medicine, University of Sydney, Sydney, Australia; ${ }^{3}$ Genetic Health Queensland, Royal Brisbane and Women's Hospital, Brisbane, Australia; ${ }^{4}$ Victorian Clinical Genetics Service, Murdoch Childrens Research Institute, Melbourne, Australia; ${ }^{5}$ Genetic Medicine Department, Royal Melbourne Hospital, Melbourne, Australia; ${ }^{6}$ Department of Cardiology, Royal Brisbane and Women's Hospital, Brisbane, Australia; ${ }^{7}$ School of Medicine, University of Queensland, Brisbane, Australia; ${ }^{8}$ Department of Cardiology, Royal Prince Alfred Hospital, Sydney, Australia. Correspondence: Christopher Semsarian (c.semsarian@centenary.org.au)
} 
management perspective and in health-economic terms. This study sought to identify clinical variables that may predict a positive genetic test result in probands with HCM attending a specialized cardiac genetic clinic.

\section{MATERIALS AND METHODS Patient cohort}

Consecutive probands with HCM attending one of three major specialized cardiac genetic clinics in Australia over a 10-year period (2002-2011) or who were enrolled in the Australian Genetic Heart Disease Registry ${ }^{16}$ were included. Those meeting the eligibility criteria were included, specifically patients with a clinical diagnosis of $\mathrm{HCM}^{17}$ and who had undergone mutation detection testing. Probands not meeting clinical diagnostic criteria for HCM were not included (i.e., physiologic hypertrophy in response to athletic training or hypertension). Only the proband was included in the analysis, and for the purpose of this study the proband was defined as the family member with clinical HCM selected to undergo initial genetic testing to identify the causative mutation in his or her family.

\section{Clinical evaluation}

All probands underwent routine clinical evaluation including clinical history, physical examination, 12-lead electrocardiography, and 2D and M-mode transthoracic echocardiogram. Clinical variables included age at diagnosis, occurrence of an out-of-hospital cardiac arrest (OHCA), or SCD of the proband either before or subsequent to genetic testing, and key echocardiographic parameters including maximum left-ventricular wall thickness (LVWT).

Family history information was collected directly from the patient by a cardiac genetic counselor or clinical geneticist and confirmed by medical record where possible, as part of the clinical assessment carried out in the specialized multidisciplinary clinics. ${ }^{18,19}$ In all cases, clinical surveillance of first-degree relatives was encouraged and most often carried out in the same center. A positive family history of disease included those with an established family history of HCM with two or more family members having a clinical diagnosis of HCM. A negative family history was defined as no additional cases of clinically apparent HCM in a family, i.e., sporadic HCM. Family history of SCD was determined on the basis of information provided by the family and/or confirmatory medical reports (i.e., postmortem examination). Only deaths for which the underlying cause was probable or definite HCM were included.

\section{Genetic testing}

Genetic testing was performed as part of the clinical service by a number of different genetic testing laboratories. All patients had 8-10 HCM genes screened (MYBPC3, MYH7, TPM1, TNNT2, TNNI3, ACTC1, MYL2, MYL3, ACTN2, TCAP) by a range of methods including single-stranded conformation polymorphism analysis, and direct DNA sequencing as previously described..$^{20,21}$ This reflects the evolution over the past decade of genetic testing strategies in a clinic setting. Probands with an identified gene mutation were classed as a positive genetic result, whereas those with no identified causative mutation were categorized as an indeterminate genetic result. Cases for which the pathogenicity of a variant could not be determined, i.e., a variant of uncertain significance, were deemed indeterminate genetic results. Pathogenicity was assessed on the basis of the following criteria, as previously described: (i) a nonsynonymous variant that causes an amino acid change that is conserved among species; (ii) the variant is not present in healthy control populations, including the 1000 Genomes and dbSNP databases with a minor allele frequency of $<0.01$; (iii) there is cosegregation with affected family members; and/or (iv) it has been previously reported as a causative HCM mutation..$^{22,23}$ This information was evaluated by the attending cardiologist and/or clinical geneticists on a case-by-case basis as part of the clinical service, and accordingly used for predictive testing where indicated.

\section{Statistical analysis}

Data were analyzed using Prism (version 5.0) and SPSS Statistics version 20 (SPSS, Chicago, IL). Clinical predictors of a genetic diagnosis were assessed using unpaired $t$-tests, $\chi^{2}$ analysis, and one-way analysis of variance. Single-factor and multiple logistic regression analyses were used to assess variables associated with a positive genetic result following stepwise selection for modelfit. A $P$ value of $<0.05$ was considered statistically significant.

\section{RESULTS}

\section{Clinical and genetic features in probands with HCM}

A total of 265 probands with HCM were included in the study. The mean age of probands was $51 \pm 18$ years and $164(62 \%)$ were males. The mean age at diagnosis was $41 \pm 18$ years, and mean maximum LVWT was $21 \pm 6 \mathrm{~mm}$. There were 141 (53\%) probands with an established family history of disease, including $65(25 \%)$ with a family history of SCD. Twenty-six (10\%) probands had suffered an OHCA or had died suddenly, before or subsequent to genetic testing.

Table 1 shows the outcomes from genetic testing in HCM probands. A genetic diagnosis was made in 138 (52\%) probands, whereas 127 (48\%) received an indeterminate genetic result. A variant of uncertain significance was identified in six (2\%) patients, and these probands were classified as having an indeterminate genetic result. The group with mutations identified were younger $(P<0.0001)$, were diagnosed at a younger age $(P<0.0001)$, had more severe $\operatorname{LVWT}(P=0.03)$, and were significantly more likely to be female $(P=0.005)$. The frequency distribution of LVWT between probands with positive and those with indeterminate genetic results highlights the fact that either group can show minimal to severe LVWT (Supplementary Figure S1 online).

The distribution of disease genes identified in the cohort is shown in Supplementary Table S1 online. Mutations in $10 \mathrm{HCM}$ genes were identified, with MYBPC3 and MYH7 accounting for more than $50 \%$ of cases. Multiple mutation genotypes were present in $16 \mathrm{HCM}$ probands (6\%) and included a 
Table 1 Characteristics of the cohort by gene result

\begin{tabular}{|c|c|c|c|c|}
\hline \multirow[b]{2}{*}{ Characteristic } & \multirow[b]{2}{*}{ Total cohort } & \multicolumn{2}{|c|}{ Gene result } & \multirow[b]{2}{*}{$P$ value } \\
\hline & & Positive & No mutation ID & \\
\hline$n(\%)$ & 265 & $138(52)$ & $127(48)$ & \\
\hline Male (\%) & $164(62)$ & $74(54)$ & $90(70)$ & 0.005 \\
\hline Age at diagnosis (years \pm SD) & $41 \pm 18$ & $34 \pm 17$ & $44 \pm 18$ & $<0.0001$ \\
\hline Family history of HCM (\%) & $141(53)$ & $102(74)$ & $39(31)$ & $<0.0001$ \\
\hline Family history SCD (\%) & $65(25)$ & $57(41)$ & $8(6)$ & 0.0003 \\
\hline
\end{tabular}

HCM, hypertrophic cardiomyopathy; ID, identified; Max LVWT, maximum left-ventricular wall thickness; NS, not significant; OHCA, out-of-hospital cardiac arrest; SCD, sudden cardiac death.

previously described triple-mutation genotype $\mathrm{e}^{24}$ and a homozygous TNNI3 mutation (Supplementary Table S2 online).

\section{Clinical variables predicting a positive genetic result}

Family history of disease was found to be an important predictor, with 102 (72\%) probands with an established family history of HCM receiving a positive genetic result, as compared with 36 (29\%) probands with no family history $(P<0.0001)$. In those probands with a family history of HCM, those who also had a positive family history of SCD were significantly more likely to have a mutation identified ( 89 vs. $59 \%, P<0.0001$ ), as shown in Table 2 and Figure 1. Univariate analysis identified age (odds ratio 0.97 ; $95 \%$ confidence interval, $0.96-0.98, P<0.0001$ ), age at diagnosis (odds ratio 0.97 ; $95 \%$ confidence interval, $0.95-$ $0.98, P<0.0001$ ), and maximum LVWT (odds ratio 1.06; $95 \%$ confidence interval 1.0-1.1, $P=0.028$ ) as also being associated with an increased likelihood of a positive genetic result.

Multivariate analysis identified many of these variables as remaining significantly associated with an increased chance of a positive genetic result, explaining $41 \%$ of the variance (Table 2 ). Age of the proband did not remain significant and was removed from the model; however, female gender, maximal LVWT, family history of HCM, and family history of SCD were significant. Age at diagnosis was borderline nonsignificant at $P=0.052$.

The gender of the proband was important in predicting a positive genetic result. Females were significantly more likely to have a mutation identified ( 63 vs. $45 \%, P=0.0052$ ). This association could be further clarified if family history of HCM was considered. Specifically, in the probands with no established family history, females were significantly more likely to have genetic diagnosis made ( 41 vs. $24 \%, P=0.0051$ ), and in the family history group, there was no difference in mutation detection rate by gender (77 vs. $69 \%, P=$ not significant) (Figure 2).

\section{Genotype-phenotype correlation}

Clinical variables were compared among probands with a causative mutation in the most common gene groups, i.e., $M Y B P C 3$, MYH7, TNNT2, ACTC2, and TNNI3. The MYH7 mutation carriers were significantly younger ( $42 \pm 14$ years vs. $52 \pm 16$ years,
Table 2 Genetic testing mutation identification rates by family history and gender, and multivariate predictors of a positive genetic result

\begin{tabular}{|c|c|c|c|}
\hline Variable & $n$ & $\%$ & $P$ value \\
\hline \multicolumn{4}{|l|}{ Family history } \\
\hline $\begin{array}{l}\text { No family history/ } \\
\text { sporadic disease }\end{array}$ & 36 & $29 \%$ & \multirow{2}{*}{$<0.0001$} \\
\hline Family history of HCM & 102 & $72 \%$ & \\
\hline $\begin{array}{l}\text { Positive family history } \\
\text { of SCD }\end{array}$ & 56 & $89 \%$ & \multirow{2}{*}{$<0.0001$} \\
\hline $\begin{array}{l}\text { No known SCD in } \\
\text { family }\end{array}$ & 44 & $59 \%$ & \\
\hline \multicolumn{4}{|l|}{ Gender } \\
\hline Male proband & 74 & $45 \%$ & \multirow{2}{*}{0.005} \\
\hline Female proband & 64 & $63 \%$ & \\
\hline $\begin{array}{l}\text { Predictor of positive } \\
\text { result }\end{array}$ & $\begin{array}{l}\text { Adjusted } \\
\text { OR }\end{array}$ & $95 \% \mathrm{Cl}$ & P value \\
\hline Age at diagnosis & 0.98 & $0.96-1.00$ & 0.056 \\
\hline Female gender & 2.30 & $1.13-4.67$ & 0.021 \\
\hline Maximal LVWT & 1.09 & $1.02-1.16$ & 0.009 \\
\hline Family history of $\mathrm{HCM}$ & 4.07 & $1.86-8.89$ & 0.003 \\
\hline Family history of SCD & 4.30 & $1.62-11.38$ & 0.022 \\
\hline
\end{tabular}

$P=0.0035)$, and diagnosed earlier in life as compared with the MYBPC 3 mutation carriers ( $27 \pm 15$ years vs. $40 \pm 17$ years, $P$ $=0.0036$ ) (Table 3). TNNT2 mutation carriers had a younger age at diagnosis, and TNNI3 mutation carriers were more likely to have had an OHCA or SCD, but these trends did not reach statistical significance.

\section{Multiple-mutation genotypes}

The clinical and genetic characteristics of the probands with multiple mutations are shown in Supplementary Table S2 online. MYBPC3 and MYH7 were the two most common genes involved in these multiple-mutation probands, which 


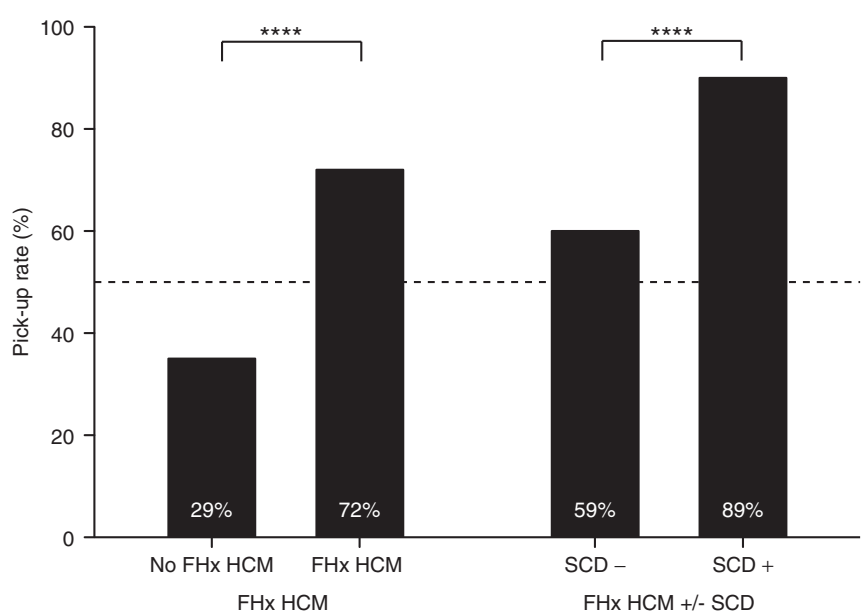

Figure 1 Mutation detection rates by family history. There was a significantly greater chance of identifying a mutation in those probands with an established family history ( $\mathrm{FHx}$ ) of hypertrophic cardiomyopathy (HCM) (72 vs. $29 \%, P<0.0001$ ) and within the positive family history group; a history of sudden cardiac death (SCD) further increased the detection rate ( 89 vs. $59 \%, P<0.0001$ ). The line at $50 \%$ highlights the quoted mutation detection rate as told to probands before genetic testing. ${ }^{*} * * P=0.0001$.

included compound heterozygotes (different mutations in the same gene), double heterozygotes (a mutation in two different genes), and one individual who was homozygous for a TNNI3 mutation. Comparison of clinical variables between singleand multiple-mutation carriers showed a marked increase in the occurrence of an OHCA or SCD in probands, with 5 of 16 (31\%) multiple mutation carriers suffering an OHCA or SCD as compared with 9 of $122(7 \%)$ in single-gene mutation carriers $(P=0.0121)$ (Table 3$)$.

\section{DISCUSSION}

This study identified family history as a key clinical predictor of a positive genetic result in probands with HCM. The difference in mutation detection rate was almost threefold higher if a family history of HCM was present as compared with no family history. This mutation detection rate was further increased when probands also had a history of HCM-related sudden death. This important finding has direct clinical relevance for informing patients about their specific individual likelihood for identification of an HCM-causing gene mutation on the basis of their family history.

In current clinical practice, the availability of genetic testing in HCM is primarily limited to major centers. The cost of diagnostic genetic testing and the variable mutation detection rates have hindered the widespread availability of genetic testing in HCM. Most cardiologists will inform their probands with HCM that the chance of identifying a causative mutation from current genetic testing is $50 \%$. The current study identified a simple parameter, i.e., the taking of a detailed and thorough family history at the time of clinical evaluation, as an important measure in predicting those patients who may have a positive gene result. The differences are surprisingly large. The mutation detection rate for those with a family history of HCM was
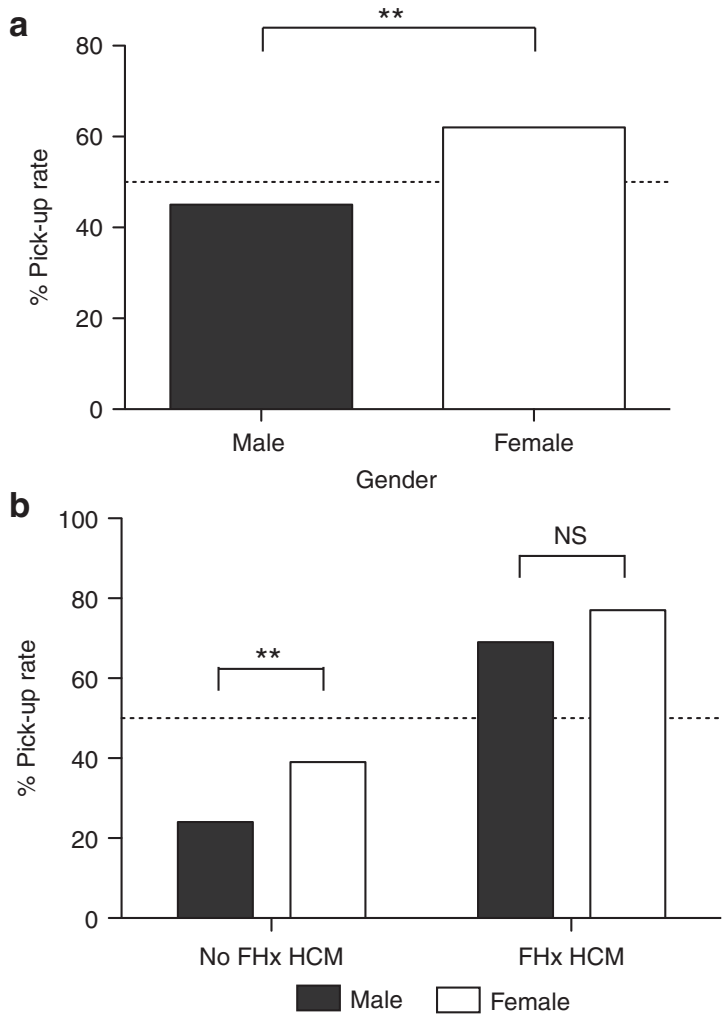

Figure 2 Mutation detection rates by gender and family history. Female gender of the proband was found to be significantly associated with an increased chance of a positive genetic result (63 vs. $45 \%, P=0.005$ ). When grouped by family history of disease, the gender association was not significant in those with a family history of disease (69 vs. $77 \%, P=$ not significant (NS)). In those probands with no family history of disease, female gender was still significantly associated with a greater chance of a positive genetic result (41 vs. $24 \%, P=0.005)$. ${ }^{*} P=0.005$.

$72 \%$ and if they also had a history of SCD, this rate increased to $89 \%$. Indeed, multivariate analysis identified the combination of female gender, increased LVWT, and family history of HCM and SCD as being most predictive of identifying a pathogenic mutation. These data will enable cardiologists and clinical geneticists to inform their patients better of the chances of a positive genetic test result based on their specific clinical family history. In those probands in whom there is a low chance of obtaining a positive genetic result (29\%), this will provide clarity on the likelihood of a mutation being identified. It is important to highlight that genetic testing is encouraged in all cases, even when the mutation detection rate is low. Cost-effectiveness analysis in this setting supports this idea, with our group previously reporting sensitivity analysis of proband mutation detection rates of $40-70 \%$ remaining very cost effective ( $40 \%$ mutation detection rate; ICER (incremental cost effectiveness ratio) \$A1601 per quality adjusted life year gained). ${ }^{13}$

The key clinical predictor of a positive genetic result was found to be an established family history of HCM. Two previous studies, in HCM populations in the United States and Germany, support the finding that a positive family history of HCM results in a higher mutation detection rate, although the 
Table 3 Cohort characteristics by genotype

\begin{tabular}{|c|c|c|c|c|c|c|c|c|}
\hline & МУВРСЗ & MYH7 & TNNT2 & ACTN2 & TNNI3 & Single & Multiple & $P$ value \\
\hline$n(\%)$ & $53(20)$ & $44(17)$ & $7(3)$ & $5(2)$ & $5(2)$ & $122(46)$ & $16(6)$ & - \\
\hline Mean age (years) & $52 \pm 16^{a}$ & $42 \pm 14^{a}$ & $46 \pm 11$ & $47 \pm 19$ & $44 \pm 22$ & $48 \pm 16$ & $40 \pm 19$ & $0.0035^{a}$ \\
\hline Age at diagnosis (years) & $40 \pm 17^{a}$ & $27 \pm 15^{a}$ & $26 \pm 17$ & $41 \pm 18$ & $31 \pm 25$ & $35 \pm 17$ & $31 \pm 17$ & $0.0036^{a}$ \\
\hline Max LVWT (mm) & $23 \pm 7$ & $23 \pm 5$ & $23 \pm 6$ & $23 \pm 8$ & $19 \pm 5$ & $22 \pm 6$ & $22 \pm 7$ & NS \\
\hline Family history of SCD & $24(44)$ & $20(45)$ & $4(57)$ & $1(20)$ & $3(60)$ & $52(43)$ & $5(31)$ & NS \\
\hline
\end{tabular}

HCM, hypertrophic cardiomyopathy; Max LVWT, maximum left-ventricular wall thickness; NS, not significant; OHCA, out-of-hospital cardiac arrest; SCD, sudden cardiac death; single, single-mutation carrier; multiple, multiple-mutation carrier. ${ }^{a}$ Statistically significant values.

increases were less substantial (54 and 68\%, respectively).,10 This further highlights the need for a detailed family history and pedigree to be taken. Although the attending cardiologist can obtain such a history, in the setting of a multidisciplinary clinic, a cardiac genetic counselor or clinical geneticist experienced in this area is more likely to obtain a detailed and thorough history and to have the capacity to obtain confirmatory medical data related to sudden deaths in the family. ${ }^{18}$ In addition, the capacity of genetic counselors to assist in coordinating clinical surveillance of family members to identify presymptomatic disease is critical.

Additional variables found to predict a positive genetic result were the young age of the proband at clinical evaluation, an earlier age at diagnosis, gender, and increased maximal LVWT. Although these variables were shown to be significantly associated with a positive genetic result, their clinical applicability is limited. The frequency distribution of maximal LVWT by positive or indeterminate genetic result highlighted the fact that patients from either group can have a wall thickness at either end of the spectrum, from mild to severe (i.e., $30 \mathrm{~mm}$ and above). Furthermore, the mean maximum LVWT differed between the groups by $<2 \mathrm{~mm}$, which although statistically significant, would have limited value as a predictive tool in the clinical setting.

The influence of gender in genetic testing outcomes in our HCM cohort was observed in the current study. Overall, males had a lower mutation detection rate than females, with a disproportionate number of males populating the indeterminate genetic result group. When adjusted for a number of variables, the combination of male gender, reduced LVWT, and absence of family history of HCM or SCD was least likely to yield a causative mutation. Of note, in those with no family history, female gender significantly increased the likelihood of a genetic diagnosis, whereas there was no gender association in those probands with a family history of HCM (i.e., males or females were not more likely to have a mutation identified). Understanding the underlying clinical and possible genetic basis of these male patients with apparent sporadic HCM will be an important consideration and may represent nonheritable phenocopies of HCM.
Interesting gene-specific disease characteristics were observed in the current study. Although initial studies supported the notion of mutation-specific clinical outcomes, ${ }^{25}$ subsequent studies ${ }^{26}$ and the identification of the vast clinical and genetic heterogeneity in HCM have limited the clinical utility of genotype in guiding management. ${ }^{1,27}$ In the current study, MYH7 mutation carriers were found to be significantly younger, and they presented at a younger age, as compared with MYBPC3 mutation carriers. Mutations in specific genes did not correlate significantly with clinical features, but the number of gene mutations correlated with some measures of disease severity, specifically the occurrence of an OHCA or SCD in the proband. Our group ${ }^{20,22}$ and others, ${ }^{8,9,28}$ have previously suggested that multiple-mutation carriers express a more severe phenotype, including younger age at diagnosis, increased LVWT, and a higher incidence of sudden death events. ${ }^{29}$ The current data further support the link between the number of disease-causing mutations and clinical severity of disease, and may ultimately lead to using this information as part of risk-stratification algorithms in assessing the risk of developing heart failure and sudden death in HCM.

The small number of HCM probands in our cohort who had a family history but in whom no causative gene mutation was identified represent a tantalizing subgroup for further genetic evaluation. Only 7 of 63 (11\%) probands with an established family history of HCM and SCD failed to have a causative mutation identified. These seven probands therefore have clinical HCM, have an inherited disease due to the family history, but do not harbor a known HCM-causing gene mutation. These patients may have one of the rare disease genes not tested in our cohort, or may have an as yet unidentified disease gene. With the rapid evolution of next-generation sequencing technologies, genome-wide genetic analysis approaches are likely to identify new causative genes in HCM.

\section{Study limitations}

This study presents a description of the clinical experience of the three major specialized cardiac genetic clinics in Australia over a period of 10 years. Therefore, the method of genetic testing employed for each case was dependent on the best available 
technology at the time and so incorporates a number of different genetic testing approaches. Family history information was obtained both from a detailed family history taken from the proband as well as extensive and thorough clinical family screening that was initiated as part of routine clinical management in relatives. Every effort was made to obtain all possible family history information in each case.

\section{Conclusion}

Family history of disease is an important clinical predictor of identifying a pathogenic mutation following HCM genetic testing. Additional information, such as gender, maximal LVWT, and family history of SCD, adds further to the likelihood of having a positive genetic result. These findings have direct clinical relevance for informing patients about their specific individual likelihood for identification of an HCM-causing gene mutation on the basis of their family history. Furthermore, by determining the cost-effectiveness of genetic testing, this may guide health policy and reimbursement decisions. The multidisciplinary specialized clinic, incorporating cardiology and clinical genetics, is highlighted as an ideal model for the optimal and most comprehensive care of patients with HCM and their families.

\section{SUPPLEMENTARY MATERIAL}

Supplementary material is linked to the online version of the paper at http://www.nature.com/gim

\section{ACKNOWLEDGMENTS}

J.I. is the recipient of a cofunded National Heart Foundation and National Health and Medical Research Council (NHMRC) Early Career Fellowship, and C.S. is the recipient of a NHMRC Practitioner Fellowship. This study was also supported, in part, by an NHMRC project grant.

\section{DISCLOSURE}

The authors declare no conflict of interest.

\section{REFERENCES}

1. Seidman JG, Seidman C. The genetic basis for cardiomyopathy: from mutation identification to mechanistic paradigms. Cell 2001;104:557-567.

2. Maron BJ, Maron MS, Semsarian C. Genetics of hypertrophic cardiomyopathy after 20 years: clinical perspectives. J Am Coll Cardiol 2012;60:705-715.

3. Maron BJ. Sudden death in young athletes. N Engl J Med 2003;349:10641075.

4. Maron BJ, Gardin JM, Flack JM, Gidding SS, Kurosaki TT, Bild DE. Prevalence of hypertrophic cardiomyopathy in a general population of young adults. Echocardiographic analysis of 4111 subjects in the CARDIA Study. Coronary Artery Risk Development in (Young) Adults. Circulation 1995;92: 785-789.

5. Ackerman MJ, Priori SG, Willems S, et al. HRS/EHRA expert consensus statement on the state of genetic testing for the channelopathies and cardiomyopathies this document was developed as a partnership between the Heart Rhythm Society (HRS) and the European Heart Rhythm Association (EHRA). Heart Rhythm 2011;8:1308-1339.

6. Lind JM, Chiu C, Semsarian C. Genetic basis of hypertrophic cardiomyopathy. Expert Rev Cardiovasc Ther 2006;4:927-934

7. Van Driest SL, Ommen SR, Tajik AJ, Gersh BJ, Ackerman MJ. Yield of genetic testing in hypertrophic cardiomyopathy. Mayo Clin Proc 2005;80:739-744.
8. Van Driest SL, Ommen SR, Tajik AJ, Gersh BJ, Ackerman MJ. Sarcomeric genotyping in hypertrophic cardiomyopathy. Mayo Clin Proc 2005;80:463469.

9. Richard P, Charron P, Carrier L, et al.; EUROGENE Heart Failure Project. Hypertrophic cardiomyopathy: distribution of disease genes, spectrum of mutations, and implications for a molecular diagnosis strategy. Circulation 2003;107:2227-2232.

10. Erdmann J, Daehmlow S, Wischke S, et al. Mutation spectrum in a large cohort of unrelated consecutive patients with hypertrophic cardiomyopathy. Clin Genet 2003;64:339-349.

11. Gray B, Ingles J, Semsarian C. Natural history of genotype positivephenotype negative patients with hypertrophic cardiomyopathy. Int I Cardiol 2011;152:258-259.

12. Maron BJ, Yeates L, Semsarian C. Clinical challenges of genotype positive (+)-phenotype negative (-) family members in hypertrophic cardiomyopathy. Am J Cardiol 2011;107:604-608.

13. Ingles J, McGaughran J, Scuffham PA, Atherton J, Semsarian C. A costeffectiveness model of genetic testing for the evaluation of families with hypertrophic cardiomyopathy. Heart 2012;98:625-630.

14. Wordsworth S, Leal J, Blair E, et al. DNA testing for hypertrophic cardiomyopathy: a cost-effectiveness model. Eur Heart J 2010;31:926-935.

15. Andersen PS, Havndrup O, Hougs L, et al. Diagnostic yield, interpretation, and clinical utility of mutation screening of sarcomere encoding genes in Danish hypertrophic cardiomyopathy patients and relatives. Hum Mutat 2009;30:363370.

16. Ingles J, McGaughran J, Vohra J, et al. Establishment of an Australian National Genetic Heart Disease Registry. Heart Lung Circ 2008;17:463-467.

17. Gersh BJ, Maron BJ, Bonow RO, et al.; American College of Cardiology Foundation/American Heart Association Task Force on Practice Guidelines; American Association for Thoracic Surgery; American Society of Echocardiography; American Society of Nuclear Cardiology; Heart Failure Society of America; Heart Rhythm Society; Society for Cardiovascular Angiography and Interventions; Society of Thoracic Surgeons. 2011 ACCF/AHA guideline for the diagnosis and treatment of hypertrophic cardiomyopathy: a report of the American College of Cardiology Foundation/American Heart Association Task Force on Practice Guidelines. Circulation 2011;124:e783e831.

18. Ingles J, Yeates $L$, Semsarian $C$. The emerging role of the cardiac genetic counselor. Heart Rhythm 2011;8:1958-1962.

19. Ingles J, Zodgekar PR, Yeates L, Macciocca I, Semsarian C, Fatkin D; CSANZ Cardiac Genetic Diseases Council Writing Group. Guidelines for genetic testing of inherited cardiac disorders. Heart Lung Circ 2011;20:681-687.

20. Ingles J, Doolan A, Chiu C, Seidman J, Seidman C, Semsarian C. Compound and double mutations in patients with hypertrophic cardiomyopathy: implications for genetic testing and counselling. J Med Genet 2005;42:e59.

21. Chiu C, Bagnall RD, Ingles J, et al. Mutations in alpha-actinin-2 cause hypertrophic cardiomyopathy: a genome-wide analysis. J Am Coll Cardiol 2010;55:1127-1135.

22. Maron BJ, Semsarian C. Prevention of sudden death for patients with cardiomyopathies another step forward. J Am Coll Cardiol 2012;59: 501-502.

23. Chiu C, Tebo M, Ingles J, et al. Genetic screening of calcium regulation genes in familial hypertrophic cardiomyopathy. J Mol Cell Cardiol 2007;43: 337-343.

24. Girolami F, Ho CY, Semsarian C, et al. Clinical features and outcome of hypertrophic cardiomyopathy associated with triple sarcomere protein gene mutations. J Am Coll Cardiol 2010;55:1444-1453.

25. Watkins H, Rosenzweig A, Hwang DS, et al. Characteristics and prognostic implications of myosin missense mutations in familial hypertrophic cardiomyopathy. N Engl J Med 1992;326:1108-1114.

26. Semsarian C, Yu B, Ryce C, Lawrence C, Washington H, Trent RJ. Sudden cardiac death in familial hypertrophic cardiomyopathy: are "benign" mutations really benign? Pathology 1997;29:305-308.

27. Saltzman AJ, Mancini-DiNardo D, Li C, et al. Short communication: the cardiac myosin binding protein C Arg502Trp mutation: a common cause of hypertrophic cardiomyopathy. Circ Res 2010;106:1549-1552.

28. Van Driest SL, Vasile VC, Ommen SR, et al. Myosin binding protein C mutations and compound heterozygosity in hypertrophic cardiomyopathy. J Am Coll Cardio/ 2004;44:1903-1910.

29. Kelly M, Semsarian C. Multiple mutations in genetic cardiovascular disease: a marker of disease severity? Circ Cardiovasc Genet 2009;2:182-190. 